\title{
Field theory of massive and massless vector particles in the Duffin-Kemmer-Petiau formalism
}

\author{
S. I. Kruglov \\ University of Toronto at Scarborough, \\ Physical and Environmental Sciences Department, \\ 1265 Military Trail, Toronto, Ontario, Canada M1C 1A4
}

\begin{abstract}
Field theory of massive and massless vector particles is considered in the first-order formalism. The Hamiltonian form of equations is obtained after the exclusion of non-dynamical components. We obtain the canonical and symmetrical Belinfante energy-momentum tensors and their nonzero traces. We note that the dilatation symmetry is broken in the massive case but in the massless case the modified dilatation current is conserved. The canonical quantization is performed and the propagator of the massive fields is found in the Duffin-Kemmer-Petiau formalism.
\end{abstract}

\section{Introduction}

The theory of the unified weak and electromagnetic interaction between elementary particles including vector particles (Standard Model) is renormalized field theory [1]. The crucial role plays the spontaneous breaking symmetry when massless vector fields acquire masses due to the Higgs mechanism. Nowadays, it is of great importance for searching the scalar Higgs bosons at Large Hadronic Collider. Anyway, the old problem of describing massive and massless vector particles is of theoretical interest. It is well known that the Proca equations for vector particles can be cast into the first-order (the matrix form) Duffin-Kemmer-Petiau (DKP) relativistic wave equation (RWE) [2], 3], 4], [5]. One can find early references on DKP equations in [6]. The matrix form of RWE is also convenient for the formulation of higher derivative field equations [7, 8], [9], [10], fields with multi-spin [11, [12, [13, [14, [15], [16], Einstein gravity equations [17], fields in curved space-time [18], [19], [20] and quantum chromodynamics [21]. There is a vast number of papers devoted to DKP equations, and, therefore, we mention only some part of them. 
The goal of this paper is to give the systematic description of massive and massless vector fields in the DKP formalism, to find solutions in the form of projection matrix-dyads, to obtain the quantum-mechanical Hamiltonian, to investigate the dilatation symmetry, and to perform the canonical quantization.

The paper is organized as follows. In Sec.2, we consider massive and massless vector fields in the form of RWE with two mass parameters. Solutions of the wave equation for a free massive field are obtained in the form of matrix-dyads. The quantum-mechanical Hamiltonians are found for massive and massless vector fields in Sec.3. We obtain the canonical and the symmetrical Belinfante energy momentum tensors and the dilatation current in Sec.4. It is demonstrated that the dilatation symmetry is broken for massive fields but in the massless case the modified dilatation current is conserved. In Sec.5, the canonical quantization of massive fields is performed and the propagator of fields is obtained in the DKP formalism. We draw a conclusion in Sec.6. In Appendix, we demonstrate that the system of equations with two mass parameter introduced can describe massive or massless fields.

The Euclidean metric is used and the system of units $\hbar=c=1$ is explored.

\section{Vector particles in the first-order formal- ism}

To consider the massive and massless vector fields, let us introduce the system of equations with two mass parameters

$$
\begin{gathered}
\partial_{\nu} \psi_{[\mu \nu]}+m_{1} \psi_{\mu}=0, \\
\partial_{\nu} \psi_{\mu}-\partial_{\mu} \psi_{\nu}+m_{2} \psi_{[\mu \nu]}=0 .
\end{gathered}
$$

System of equations (1) is the generalization of Proca equations, and describes massive or massless particles depending on parameters $m_{1}, m_{2}$ chosen (see Appendix). At $m_{1}=m_{2}$, after the renormalization of fields, one arrives at the Proca equations. At $m_{1}=0$, we have the massless case corresponding to the Maxwell equations. Excluding the antisymmetrical tensor $\psi_{[\mu \nu]}$ in Eq.(1) (see (A1) in Appendix), in the general case, we find the wave equation for the field $\psi_{\mu}$ possessing the mass $m=\sqrt{m_{1} m_{2}}$. Thus, Eq.(1) 
gives the convenient parametrization for description of massive or massless vector fields. The fields $\psi_{A}(A=\mu,[\mu \nu])$ have the same dimension due to the presence of the mass parameter $m_{2}$.

Introducing the wave function

$$
\Psi(x)=\left\{\psi_{A}(x)\right\}=\left(\begin{array}{c}
\psi_{\mu}(x) \\
\psi_{[\mu \nu]}(x)
\end{array}\right) \quad(A=\mu,[\mu \nu]),
$$

and the elements of the entire matrix algebra $\varepsilon^{A, B}$, with properties

$$
\left(\varepsilon^{M, N}\right)_{A B}=\delta_{M A} \delta_{N B}, \quad \varepsilon^{M, A} \varepsilon^{B, N}=\delta_{A B} \varepsilon^{M, N},
$$

where $A, B, M, N=\mu,[\mu \nu]$, the system of equations (1) can be represented in the matrix form

$$
\left[\partial_{\mu}\left(\varepsilon^{\nu,[\nu \mu]}+\varepsilon^{[\nu \mu], \nu}\right)+m_{1} \varepsilon^{\mu, \mu}+m_{2} \frac{1}{2} \varepsilon^{[\nu \mu],[\nu \mu]}\right]_{A B} \Psi_{B}(x)=0 .
$$

We imply a summation over all repeated indices. Defining the $10 \times 10$ matrices

$$
\beta_{\mu}=\varepsilon^{\nu,[\nu \mu]}+\varepsilon^{[\nu \mu], \nu}, \quad \bar{P}=\varepsilon^{\mu, \mu}, \quad P=\frac{1}{2} \varepsilon^{[\nu \mu],[\nu \mu]},
$$

Eq.(4) takes the form of the first-order RWE

$$
\left(\beta_{\mu} \partial_{\mu}+m_{1} \bar{P}+m_{2} P\right) \Psi(x)=0 .
$$

The matrices $\beta_{\mu}$ are Hermitian matrices, $\beta_{\mu}^{+}=\beta_{\mu}$. The projection operator $\bar{P}=\bar{P}^{+}$extracts the four-dimensional vector subspace $\left(\psi_{\mu}\right)$ of the wave function $\Psi$, and the projection operator $P=P^{+}$extracts the six-dimensional tensor subspace corresponding to the $\psi_{[\mu \nu]}$. The matrices $\beta_{\mu}$ obey the DKP algebra

$$
\beta_{\mu} \beta_{\nu} \beta_{\alpha}+\beta_{\alpha} \beta_{\nu} \beta_{\mu}=\delta_{\mu \nu} \beta_{\alpha}+\delta_{\alpha \nu} \beta_{\mu},
$$

and matrices $\bar{P}, P$ are projection matrices

$$
\begin{gathered}
\bar{P}^{2}=\bar{P}, \quad P^{2}=P, \quad \bar{P}+P=1, \quad \bar{P} P=P \bar{P}=0, \\
\beta_{\mu} \bar{P}+\bar{P} \beta_{\mu}=\beta_{\mu}, \quad \beta_{\mu} P+P \beta_{\mu}=\beta_{\mu} .
\end{gathered}
$$

At $m_{1}=m_{2}=m$, from Eq.(6), we arrive at the DKP equation for massive vector fields

$$
\left(\beta_{\mu} \partial_{\mu}+m\right) \Psi(x)=0
$$


For massless DKP equation, corresponding to the Maxwell equations, we put $m_{1}=0$ in Eq.(6), and arrive at

$$
\left(\beta_{\mu} \partial_{\mu}+m_{2} P\right) \Psi(x)=0 .
$$

In [22], 23] the case $m_{1}=0$, Eq.(10), was considered. It should be noted that massless DKP model describes electromagnetic fields and is invariant under a local U(1) gauge symmetry and being a fundamental requirement for their description. The gauge transformations in DKP formalism are given by

$$
\Psi^{\prime}=\Psi+\bar{P} \Phi
$$

Then with the help of Eq.(8),(10), we obtain

$$
\left(\beta_{\mu} \partial_{\mu}+m_{2} P\right) \Psi^{\prime}=(1-\bar{P}) \beta_{\mu} \partial_{\mu} \Phi .
$$

Thus Eq.(10) is invariant under gauge transformations if the function $\Phi$ obeys the equation as follows (see also[22]): $P \beta_{\mu} \partial_{\mu} \Phi=0$. It is easy to verify that this equation is valid for the function

$$
\Phi(x)=\left(\begin{array}{c}
\partial_{\mu} \Lambda(x) \\
0
\end{array}\right)
$$

which leads to the gauge transformations: $\psi_{\mu}^{\prime}(x)=\psi_{\mu}(x)+\partial_{\mu} \Lambda(x)$. The gauge invariance of massless DKP equation was also discussed in [24].

Now, we investigate the general case, Eq.(6), including two mass parameters, $m_{1}$ and $m_{2}$. The Lorentz group generators in the 10-dimension representation space are given by

$$
J_{\mu \nu}=\beta_{\mu} \beta_{\nu}-\beta_{\nu} \beta_{\mu}=\varepsilon^{[\lambda \mu],[\lambda \nu]}+\varepsilon^{\mu, \nu}-\varepsilon^{[\lambda \nu],[\lambda \mu]}-\varepsilon^{\nu, \mu},
$$

and obey the commutation relations

$$
\begin{gathered}
{\left[J_{\rho \sigma}, J_{\mu \nu}\right]=\delta_{\sigma \mu} J_{\rho \nu}+\delta_{\rho \nu} J_{\sigma \mu}-\delta_{\rho \mu} J_{\sigma \nu}-\delta_{\sigma \nu} J_{\rho \mu}} \\
{\left[\beta_{\lambda}, J_{\mu \nu}\right]=\delta_{\lambda \mu} \beta_{\nu}-\delta_{\lambda \nu} \beta_{\mu}, \quad\left[\bar{P}, J_{\mu \nu}\right]=0, \quad\left[P, J_{\mu \nu}\right]=0 .}
\end{gathered}
$$

Eq.(6) is form-invariant under the Lorentz transformations because of Eq.(12). The Lorentz-invariant is $\bar{\Psi} \Psi=\Psi^{+} \eta \Psi$, where $\Psi^{+}$is the Hermitian-conjugated wave function, and the Hermitianizing matrix, $\eta$, is given by

$$
\eta=\varepsilon^{m, m}-\varepsilon^{4,4}+\varepsilon^{[m 4],[m 4]}-\frac{1}{2} \varepsilon^{[m n],[m n]} .
$$


The $\eta$ is the Hermitian matrix, $\eta^{+}=\eta$, and obeys the relations: $\eta \beta_{m}=$ $-\beta_{m} \eta(\mathrm{m}=1,2,3), \eta \beta_{4}=\beta_{4} \eta$. With the help of these relations, one finds the "conjugated" equation

$$
\bar{\Psi}(x)\left(\beta_{\mu} \overleftarrow{\partial}_{\mu}-m_{1} \bar{P}-m_{2} P\right)=0
$$

The Lagrangian leading to Eq.(6),(14) is

$$
\begin{aligned}
\mathcal{L} & =-\frac{1}{2} \bar{\Psi}(x)\left(\beta_{\mu} \partial_{\mu}+m_{1} \bar{P}+m_{2} P\right) \Psi(x) \\
& +\frac{1}{2} \bar{\Psi}(x)\left(\beta_{\mu} \overleftarrow{\partial_{\mu}}-m_{1} \bar{P}-m_{2} P\right) \Psi(x) .
\end{aligned}
$$

In terms of fields $\psi_{A}$, the Lagrangian (15) reads

$$
\mathcal{L}=\frac{1}{2}\left(\psi_{[\rho \mu]}^{*} \partial_{\mu} \psi_{\rho}-\psi_{\rho}^{*} \partial_{\mu} \psi_{[\rho \mu]}-m_{1} \psi_{\mu}^{*} \psi_{\mu}+\frac{m_{2}}{2} \psi_{[\rho \mu]}^{*} \psi_{[\rho \mu]}\right)+\text { c.c. },
$$

where the c.c. means the complex conjugated expression; complex conjugation $*$ does not act on the metric imaginary unit $i$ of fourth components in Eq.(16), $\psi_{\mu}^{*}=\left(\psi_{m}^{*}, i \psi_{0}^{*}\right)$, and so on, and we used $\bar{\Psi}=\left(\psi_{\mu}^{*},-\psi_{[\mu \nu]}^{*}\right)$. It is easy to verify that Euler-Lagrange equations $\partial \mathcal{L} / \partial \psi_{A}-\partial_{\mu}\left(\partial \mathcal{L} / \partial \partial_{\mu} \psi_{A}\right)=0$ lead to the equations of motion Eq.(1). The Lagrangian (15) (and (16)) vanishes for fields $\psi_{A}$ obeying Eq.(1) (or Eq.(6),(14))).

\section{Solutions to the matrix equation}

Eq.(6), in the momentum space, for the positive $(+p)$ and negative $(-p)$ energies, reads

$$
\left( \pm i \widehat{p}+m_{1} \bar{P}+m_{2} P\right) \Psi( \pm p)=0
$$

where $\widehat{p}=\beta_{\mu} p_{\mu}$, and the four-momentum being $p_{\mu}=\left(\mathbf{p}, i p_{0}\right)\left(p^{2}=\mathbf{p}^{2}-p_{0}^{2}\right)$. One can verify that the matrix of equation (17)

$$
\Lambda_{ \pm}= \pm i \widehat{p}+m_{1} \bar{P}+m_{2} P
$$

obeys the "minimal" matrix equation:

$$
\left(\Lambda_{ \pm}-m_{1}\right)\left(\Lambda_{ \pm}-m_{2}\right)\left[\left(\Lambda_{ \pm}-m_{1}\right)\left(\Lambda_{ \pm}-m_{2}\right)+p^{2}\right]=0
$$


The non-trivial solutions to Eq.(17) exist if $\operatorname{det} \Lambda_{ \pm}=0$. Therefore, the eigenvalue of the matrix $\Lambda_{ \pm}$should be zero, and this requirement results to the dispersion relation

$$
p^{2}+m_{1} m_{2}=0 .
$$

Other eigenvalues of the matrix $\Lambda_{ \pm}$are $m_{1}$ and $m_{2}$, that follows from Eq.(19). On-shell, $p^{2}=-m_{1} m_{2}$, when $m_{1} \neq m_{2}$, Eq.(19) becomes

$$
\Lambda_{ \pm}\left(\Lambda_{ \pm}-m_{1}-m_{2}\right)\left(\Lambda_{ \pm}-m_{1}\right)\left(\Lambda_{ \pm}-m_{2}\right)=0 .
$$

Solutions to Eq.(17) in the form of the projection matrix [25] follow from Eq. (21):

$$
\begin{aligned}
\Pi_{ \pm} & =N\left(\Lambda_{ \pm}-m_{1}-m_{2}\right)\left(\Lambda_{ \pm}-m_{1}\right)\left(\Lambda_{ \pm}-m_{2}\right) \\
& =\mp N i \widehat{p}\left( \pm i m_{1} \widehat{p} P \pm i m_{2} \widehat{p} \bar{P}-m_{1} m_{2}\right),
\end{aligned}
$$

so that $\Lambda_{ \pm} \Pi_{ \pm}=0$, where $N$ is the normalization constant. Every column of the matrix $\Pi_{ \pm}$is the solution to Eq.(17). The projection matrix obeys the equation

$$
\Pi_{ \pm}^{2}=\Pi_{ \pm}
$$

that leads to the normalization constant

$$
N=-\frac{1}{m_{1} m_{2}\left(m_{1}+m_{2}\right)} .
$$

This can be verified with the help of Eq.(21). We notice that Eq.(21) is the minimal polynomial only for the non-degenerate case $m_{1} \neq m_{2}$. For the special case, $m_{1}=m_{2}$, we have to use the minimal matrix equation on-shell:

$$
\Lambda_{ \pm}\left(\Lambda_{ \pm}-m\right)\left(\Lambda_{ \pm}-2 m\right)=0
$$

We obtain the projection operator from Eq.(25)

$$
\Pi_{ \pm}^{D K P}=\frac{1}{2 m^{2}}\left(\Lambda_{ \pm}^{2}-3 m \Lambda_{ \pm}+2 m^{2}\right)=\frac{ \pm i \widehat{p}( \pm i \widehat{p}-m)}{2 m^{2}} .
$$

Eq.(22) at $m_{1}=m_{2}$ becomes Eq.(26) .

We use the spin projection operators [25]:

$$
S_{( \pm 1)}=\frac{1}{2} \sigma_{p}\left(\sigma_{p} \pm 1\right), \quad S_{(0)}=1-\sigma_{p}^{2} .
$$


where

$$
\sigma_{p}=-\frac{i}{2|\mathbf{p}|} \epsilon_{a b c} p_{a} J_{b c}=-\frac{i}{|\mathbf{p}|} \epsilon_{a b c} p_{a} \beta_{b} \beta_{c} .
$$

One can check the relations $\left[\Lambda_{ \pm}, \sigma_{p}\right]=0, S_{( \pm 1)}^{2}=S_{( \pm 1)}, S_{( \pm 1)} S_{(0)}=0, S_{(0)}^{2}=$ $S_{(0)},\left[\Lambda_{ \pm}, S_{( \pm 1)}\right]=0,\left[\Lambda_{ \pm}, S_{(0)}\right]=0$. The projection operators extracting solutions to Eq.(17) with spin projections $\pm 1,0$ in the form of matrix-dyads are given by

$$
\Pi_{ \pm} S_{( \pm 1)}=\Psi_{ \pm 1} \cdot \bar{\Psi}_{ \pm 1}, \quad \Pi_{ \pm} S_{(0)}=\Psi_{0} \cdot \bar{\Psi}_{0} .
$$

The matrix-dyad has the matrix elements $(\Psi \cdot \bar{\Psi})_{A B}=\Psi_{A} \bar{\Psi}_{B}$. Eq. (29) allow us to make calculations of different electrodynamics processes with vector particles in the covariant form [25].

For the case of massless vector particles (photons), the parameter $m_{1}=0$ and $p^{2}=0$. Then the matrix of equation (10) is

$$
\Lambda_{ \pm}^{(0)}= \pm i \widehat{p}+m_{2} P
$$

and satisfies the minimal matrix equation

$$
\Lambda_{ \pm}^{(0) 2}\left(\Lambda_{ \pm}^{(0) 2}-m_{2}\right)^{2}=0
$$

In this case zero eigenvalues of the operator $\Lambda_{ \pm}^{(0)}$ are degenerated and it is impossible to obtain solutions in the form of projection matrix-dyads [25], [23. In the case of generalized Maxwell equations such difficulty is absent [26], [27].

\section{The Hamiltonian form of equations}

\subsection{Massive fields}

It is very useful to obtain the quantum mechanical Hamiltonian corresponding to equations (1) (or (6)) because the non-dynamical components will be absent. There was a suggestion in [28] to couple the electromagnetic field in the DKP equation only at the level of the Hamiltonian form. Some aspects of Hamiltonian form of DKP equations were considered in [29], [30], [31]. To exclude the non-dynamical components, we rewrite Eq.(1) in the form of two systems

$$
m_{2} \psi_{[4 m]}=\partial_{4} \psi_{m}-\partial_{m} \psi_{4}, \quad \partial_{4} \psi_{[m 4]}+\partial_{n} \psi_{[m n]}+m_{1} \psi_{m}=0
$$




$$
m_{2} \psi_{[m n]}=\partial_{m} \psi_{n}-\partial_{n} \psi_{m}, \quad \partial_{n} \psi_{[4 n]}+m_{1} \psi_{4}=0 .
$$

Equations (32) contain derivatives on the time of the dynamical components $\psi_{m}, \psi_{[m 4]}$, and Eq.(33) possess only spatial derivatives on auxiliary (non-dynamical) components $\psi_{4}, \psi_{[m n]}$. Replacing non-dynamical components from Eq.(33) into Eq.(32), we arrive at the equations for the dynamical components

$$
\begin{gathered}
i \partial_{t} \psi_{m}=-m_{2} \psi_{[4 m]}+\frac{1}{m_{1}} \partial_{m} \partial_{n} \psi_{[4 n]}, \\
i \partial_{t} \psi_{[m 4]}=\frac{1}{m_{2}}\left(\partial_{m} \partial_{n} \psi_{n}-\partial_{n}^{2} \psi_{m}\right)+m_{1} \psi_{m} .
\end{gathered}
$$

We can represent Eq.(34) in the matrix form introducing the six-component wave function

$$
\Phi(x)=\left(\begin{array}{c}
\psi_{n}(x) \\
\psi_{[m 4]}(x)
\end{array}\right) .
$$

With the help of the elements of the matrix algebra Eq.(3), we rewrite Eq.(34) in the Schrödinger-like form

$$
\begin{gathered}
i \partial_{t} \Phi(x)=\left[m_{1} \varepsilon^{[m 4], m}-m_{2} \varepsilon^{n,[4 n]}+\frac{1}{m_{1}} \varepsilon^{n,[4 m]} \partial_{n} \partial_{m}\right. \\
\left.+\frac{1}{m_{2}}\left(\varepsilon^{[m 4], n} \partial_{m} \partial_{n}-\varepsilon^{[m 4], m} \partial_{n}^{2}\right)\right] \Phi(x),
\end{gathered}
$$

where the Hamiltonian is given by

$$
\begin{gathered}
\mathcal{H}=m_{1} \varepsilon^{[m 4], m}-m_{2} \varepsilon^{n,[4 n]} \\
+\frac{1}{m_{1}} \varepsilon^{n,[4 m]} \partial_{n} \partial_{m}+\frac{1}{m_{2}}\left(\varepsilon^{[m 4], n} \partial_{m} \partial_{n}-\varepsilon^{[m 4], m} \partial_{n}^{2}\right) .
\end{gathered}
$$

We have implied that $m_{1} \neq 0, m_{2} \neq 0$. The Hamiltonian (37) is simplified for the choice $m_{1}=m_{2} \equiv m$ :

$$
\mathcal{H}=m\left(\varepsilon^{[m 4], m}-\varepsilon^{n,[4 n]}\right)+\frac{1}{m}\left[\left(\varepsilon^{n,[4 m]}+\varepsilon^{[m 4], n}\right) \partial_{m} \partial_{n}-\varepsilon^{[m 4], m} \partial_{n}^{2}\right] .
$$

The six-component wave function (35) corresponds to three spin states with positive (for particles) and negative (for antiparticles) energies and does not contain auxiliary components. We can introduce the minimal interaction of vector particles with electromagnetic fields by the replacement $\partial_{\mu} \rightarrow \partial_{\mu}-i e A_{\mu}$, where $A_{\mu}$ is the vector-potential of electromagnetic fields. 
One may verify using the properties (3) that the matrix Hamiltonian (37) in the momentum space obeys the minimal equation

$$
\left(\mathcal{H}^{2}-\mathbf{p}^{2}-m_{1} m_{2}\right)\left(\mathcal{H}^{2}+\mathbf{p}^{2}-m_{1} m_{2}\right)=0 .
$$

Equation (39) is an operator identity because in the momentum space, $\partial_{\mu} \rightarrow$ $i p_{\mu}$, the Hamiltonian is the matrix operator. If $m_{1}>0$ and $m_{2}>0$, the physical eigenvalue of the Hamiltonian squared follows from Eq.(39): $p_{0}^{2}=$

$\mathbf{p}^{2}+m_{1} m_{2}$. The projection operator extracting states with positive $\left(p_{0}\right)$ and negative $\left(-p_{0}\right)$ energies is given by

$$
\Sigma_{ \pm}= \pm \frac{1}{4 p_{0} \mathbf{p}^{2}}\left(\mathcal{H} \pm p_{0}\right)\left(\mathcal{H}^{2}+\mathbf{p}^{2}-m_{1} m_{2}\right)
$$

so that $\Sigma_{ \pm}^{2}=\Sigma_{ \pm}, \Sigma_{ \pm} \mathcal{H}=\mathcal{H} \Sigma_{ \pm}= \pm p_{0} \Sigma_{ \pm}$, where $p_{0}=\sqrt{\mathbf{p}^{2}+m_{1} m_{2}}$. The projection operator (40) allows us to get solutions to the Hamiltonian equation (36).

\subsection{Massless fields}

In the case of massless vector fields (photons), we put $m_{1}=0$ in Eq.(32),(33) and arrive at

$$
\begin{gathered}
m_{2} \psi_{[4 m]}=\partial_{4} \psi_{m}-\partial_{m} \psi_{4}, \quad \partial_{4} \psi_{[m 4]}+\partial_{n} \psi_{[m n]}=0, \\
m_{2} \psi_{[m n]}=\partial_{m} \psi_{n}-\partial_{n} \psi_{m}, \quad \partial_{n} \psi_{[4 n]}=0 .
\end{gathered}
$$

It is impossible to exclude the component $\psi_{4}$ from Eq.(41),(42). Therefore, we need to consider the $\psi_{4}$ as a dynamical component for the massless fields. To have the evolution of the $\psi_{4}$ in time, we add to Eq.(41),(42) the Lorentz condition $\partial_{m} \psi_{m}+\partial_{4} \psi_{4}=0$. After the exclusion the non-dynamical component $\psi_{[m n]}$ from Eq.(41), one finds the equations as follows:

$$
\begin{gathered}
i \partial_{t} \psi_{m}=-m_{2} \psi_{[4 m]}-\partial_{m} \psi_{4}, \\
i \partial_{t} \psi_{4}=\partial_{n} \psi_{n}, \\
i \partial_{t} \psi_{[m 4]}=\frac{1}{m_{2}}\left(\partial_{m} \partial_{n} \psi_{n}-\partial_{n}^{2} \psi_{m}\right) .
\end{gathered}
$$

Introducing the seven-component wave function

$$
\Phi_{0}(x)=\left(\begin{array}{c}
\psi_{\mu}(x) \\
\psi_{[m 4]}(x)
\end{array}\right),
$$


and using the elements of the entire matrix algebra Eq.(3), we represent Eq.(43) in the Hamiltonian form

$$
\begin{gathered}
i \partial_{t} \Phi_{0}(x)=\mathcal{H}_{0} \Phi_{0}(x) \\
\mathcal{H}_{0}=-m_{2} \varepsilon^{n,[4 n]}+\left(\varepsilon^{4, m}-\varepsilon^{m, 4}\right) \partial_{m}+\frac{1}{m_{2}}\left(\varepsilon^{[m 4], n} \partial_{m} \partial_{n}-\varepsilon^{[m 4], m} \partial_{n}^{2}\right) .
\end{gathered}
$$

With the help of Eq.(3) one can check that the Hamiltonian (45) in the momentum space satisfies the minimal equation

$$
\mathcal{H}_{0}^{2}\left(\mathcal{H}_{0}^{2}-\mathbf{p}^{2}\right)=0 .
$$

The projection operator extracting states with positive $\left(p_{0}=|\mathbf{p}|\right)$ and negative $\left(p_{0}=-|\mathbf{p}|\right)$ energies is

$$
\Sigma_{ \pm}^{0}= \pm \frac{1}{2|\mathbf{p}|^{3}}\left(\mathcal{H}_{0} \pm|\mathbf{p}|\right) \mathcal{H}_{0}^{2}
$$

and $\left(\Sigma_{ \pm}^{0}\right)^{2}=\Sigma_{ \pm}^{0}, \Sigma_{ \pm}^{0} \mathcal{H}_{0}=\mathcal{H}_{0} \Sigma_{ \pm}^{0}= \pm|\mathbf{p}| \Sigma_{ \pm}^{0}$, where $|\mathbf{p}|=\sqrt{p_{1}^{2}+p_{2}^{2}+p_{3}^{2}}$.

\section{The energy-momentum tensor and dilata- tion current}

Now, we construct the energy-momentum tensor for a given Lagrangian (15). With the help of the general equation [32]

$$
T_{\mu \nu}^{c}=\frac{\partial \mathcal{L}}{\partial\left(\partial_{\mu} \Psi(x)\right)} \partial_{\nu} \Psi(x)+\partial_{\nu} \bar{\Psi}(x) \frac{\partial \mathcal{L}}{\partial\left(\partial_{\mu} \bar{\Psi}(x)\right)}-\delta_{\mu \nu} \mathcal{L},
$$

we obtain the canonical energy-momentum tensor

$$
T_{\mu \nu}^{c}=\frac{1}{2}\left(\partial_{\nu} \bar{\Psi}(x)\right) \beta_{\mu} \Psi(x)-\frac{1}{2} \bar{\Psi}(x) \beta_{\mu} \partial_{\nu} \Psi(x),
$$

where we took into consideration that the Lagrangian (15) vanishes for fields obeying the equations of motion. With the aid of Eq.(2),(3),(5) the canonical energy-momentum tensor in the component form becomes

$$
T_{\mu \nu}^{c}=\frac{1}{2}\left(\psi_{[\rho \mu]}^{*} \partial_{\nu} \psi_{\rho}-\psi_{\rho}^{*} \partial_{\nu} \psi_{[\rho \mu]}\right)+\text { c.c. . }
$$


It is easy to verify, using Eq.(1), that the energy-momentum tensor (50) (and (49)) is conserved tensor, $\partial_{\mu} T_{\mu \nu}^{c}=0$. The canonical energy-momentum tensor is not the symmetric tensor, $T_{\mu \nu}^{c} \neq T_{\nu \mu}^{c}$, and its trace is

$$
T_{\mu \mu}^{c}=m_{1} \psi_{\mu}^{*} \psi_{\mu}-\frac{1}{2} m_{2} \psi_{[\mu \nu]}^{*} \psi_{[\mu \nu]} .
$$

One expects a nonzero trace of the energy-momentum tensor and a broken dilatation current in a massive theory. But even for massless fields, $m_{1}=$ $0, m_{2} \neq 0$, the trace of the energy-momentum tensor in (51) is non-zero. Therefore, it is of interest to investigate the scale invariance in the general case. Thus, we consider the canonical dilatation current [33]

$$
D_{\mu}^{c}=x_{\alpha} T_{\mu \alpha}^{c}+\Pi_{\mu} \mathbf{d} \Psi
$$

where

$$
\Pi_{\mu}=\frac{\partial \mathcal{L}}{\partial\left(\partial_{\mu} \Psi\right)}=-\bar{\Psi} \beta_{\mu},
$$

and the matrix $\mathbf{d}$ defines the field dimension. For the Bose fields the $\mathbf{d}$ is the unit matrix. From Eq.(52),(53), we obtain

$$
\partial_{\mu} D_{\mu}^{c}=T_{\mu \mu}^{c},
$$

where the conservation of the current was used

$$
\partial_{\mu} j_{\mu}=\partial_{\mu}\left(i \bar{\Psi} \beta_{\mu} \Psi\right)=0 .
$$

The analogous relation follows from 33]

$$
\begin{aligned}
& \partial_{\mu} D_{\mu}^{c}=\Pi_{\mu}(\mathbf{d}+1) \partial_{\mu} \Psi+\frac{\partial \mathcal{L}}{\partial \Psi} \mathbf{d} \Psi+\bar{\Psi} \mathbf{d} \frac{\partial \mathcal{L}}{\partial \bar{\Psi}}-4 \mathcal{L} \\
& =\bar{\Psi}\left(m_{1} \bar{P}+m_{2} P\right) \Psi=m_{1} \psi_{\mu}^{*} \psi_{\mu}-\frac{1}{2} m_{2} \psi_{[\mu \nu]}^{*} \psi_{[\mu \nu]} .
\end{aligned}
$$

In Eq.(56), we took into account that for the charged particles the $\Psi$ and $\bar{\Psi}$ are the independent wave functions. The dilatation symmetry is broken because of massive parameters $m_{1}$ and $m_{2}$. In the massless case, $m_{1}=0$, the dilatation current $D_{\mu}^{c}$ is also not conserved, but later we will introduce the modified conserved current. 
To obtain the symmetrical the energy-momentum, we use the expression for the Belinfante tensor [33]

$$
T_{\mu \alpha}^{B}=\frac{1}{2}\left(T_{\mu \alpha}^{c}+\partial_{\beta} X_{\beta \mu \alpha}\right),
$$

where

$$
X_{\beta \mu \alpha}=\frac{1}{2}\left[\Pi_{\beta} J_{\mu \alpha} \Psi-\Pi_{\mu} J_{\beta \alpha} \Psi-\Pi_{\alpha} J_{\beta \mu} \Psi\right]+\text { c.c. } .
$$

The additional complex conjugated term in Eq.(58) and the factor 1/2 in Eq.(57) are specific for our first-order formulation of charged fields. From Eq.(2),(5),(53), we obtain the tensor $X_{\beta \mu \alpha}$ :

$$
X_{\beta \mu \alpha}=\delta_{\alpha \beta} \psi_{\lambda}^{*} \psi_{[\lambda \mu]}-\delta_{\alpha \mu} \psi_{\lambda}^{*} \psi_{[\lambda \beta]}-\psi_{\alpha}^{*} \psi_{[\beta \mu]}+\psi_{[\mu \beta]}^{*} \psi_{\alpha}+\text { c.c. }
$$

With the help of expressions (59),(57), and equations of motion, we obtain the Belinfante symmetric energy-momentum tensor

$$
\begin{gathered}
T_{\mu \alpha}^{B}=m_{2}\left(\psi_{[\lambda \mu]} \psi_{[\alpha \lambda]}^{*}+\psi_{[\lambda \mu]}^{*} \psi_{[\alpha \lambda]}\right)-m_{1}\left(\psi_{\mu}^{*} \psi_{\alpha}+\psi_{\alpha}^{*} \psi_{\mu}\right) \\
-\frac{1}{2} \delta_{\alpha \mu} \partial_{\beta}\left(\psi_{\lambda}^{*} \psi_{[\lambda \beta]}+\psi_{[\lambda \beta]}^{*} \psi_{\lambda}\right) .
\end{gathered}
$$

From Eq.(60), one finds the trace of the energy-momentum tensor

$$
T_{\mu \mu}^{B}=2 m_{1} \psi_{\lambda}^{*} \psi_{\lambda} .
$$

In the case of massless fields, $m_{1}=0$, the trace of the Belinfante symmetric energy-momentum tensor vanishes. We note that the trace of the canonical energy-momentum tensor (51) does not equal zero for massless fields. We evaluate a modified Belinfante dilatation current 33 .

$$
D_{\mu}^{B}=x_{\alpha} T_{\mu \alpha}^{B}+V_{\mu},
$$

where the field-virial $V_{\mu}$ is given by

$$
V_{\mu}=\Pi_{\mu} \Psi-\Pi_{\alpha} J_{\alpha \mu} \Psi=-\bar{\Psi} \beta_{\mu} \Psi+\bar{\Psi} \beta_{\alpha} J_{\alpha \mu} \Psi .
$$

Using (2),(5),(11), one finds

$$
\partial_{\mu} V_{\mu}=-m_{1} \psi_{\lambda}^{*} \psi_{\lambda}-\frac{1}{2} m_{2} \psi_{[\mu \nu]}^{*} \psi_{[\mu \nu]} .
$$


As a result of Eq.(61),(62),(64), we obtain

$$
\partial_{\mu} D_{\mu}^{B}=T_{\mu \mu}^{B}+\partial_{\mu} V_{\mu}=m_{1} \psi_{\lambda}^{*} \psi_{\lambda}-\frac{1}{2} m_{2} \psi_{[\mu \nu]}^{*} \psi_{[\mu \nu]}=\partial_{\mu} D_{\mu}^{c}
$$

i.e. the same result (see Eq.(56)). For the massive fields, $m_{1} \neq 0$, the dilatation symmetry is broken as it should be. For massless fields $\left(m_{1}=0\right)$, the currents $D_{\mu}^{c}, D_{\mu}^{B}$ also are not conserved, but one can introduce the new conserved current

$$
D_{\mu}=D_{\mu}^{B}-V_{\mu}=x_{\alpha} T_{\mu \alpha}^{B}
$$

so that $\partial_{\mu} D_{\mu}=0$. Thus, massless fields (charged and neutral electromagnetic fields) possess the dilatation symmetry with the new dilatation current (66). Conformal symmetry of massless DKP equation also was investigated in another formalism in [34].

\section{The canonical quantization of massive fields}

Let us consider the massive case implying that $m_{1} \neq 0, m_{2} \neq 0$. The normalized solutions to Eq.(6) with definite energy-momentum in the form of plane waves can be written as follows:

$$
\Psi_{s}^{( \pm)}(x)=\sqrt{\frac{m_{1}+m_{2}}{2 p_{0} V}} \Psi_{s}( \pm p) \exp ( \pm i p x),
$$

where $p^{2}=\mathbf{p}^{2}-p_{0}^{2}=-m_{1} m_{2}, V$ is the normalization volume, and $s= \pm 1,0$ is the spin index. The function $\Psi_{s}( \pm p)$ obeys Eq.(17), and we explore the normalization conditions on the charge

$$
\int_{V} \bar{\Psi}_{s}^{( \pm)}(x) \beta_{4} \Psi_{s^{\prime}}^{( \pm)}(x) d^{3} x= \pm \delta_{s s^{\prime}}, \quad \int_{V} \bar{\Psi}_{s}^{( \pm)}(x) \beta_{4} \Psi_{s^{\prime}}^{(\mp)}(x) d^{3} x=0
$$

where $\bar{\Psi}_{s}^{( \pm)}(x)=\left(\Psi_{s}^{( \pm)}(x)\right)^{+} \eta$. The field operators in the second quantized theory are given by

$$
\begin{aligned}
& \Psi(x)=\sum_{p, s}\left[a_{p, s} \Psi_{s}^{(+)}(x)+b_{p, s}^{+} \Psi_{s}^{(-)}(x)\right], \\
& \bar{\Psi}(x)=\sum_{p, s}\left[a_{p, s}^{+} \bar{\Psi}_{s}^{(+)}(x)+b_{p, s} \bar{\Psi}_{s}^{(-)}(x)\right],
\end{aligned}
$$


where the positive and negative parts of the wave function $\Psi_{s}^{( \pm)}(x)$ are defined by Eq.(67). The creation and annihilation operators of particles, $a_{p, s}^{+}, a_{p, s}$ and antiparticles $b_{p, s}^{+}, b_{p, s}$ obey the usual commutation relations

$$
\begin{gathered}
{\left[a_{p, s}, a_{p^{\prime}, s^{\prime}}^{+}\right]=\delta_{s s^{\prime}} \delta_{p p^{\prime}}, \quad\left[a_{p, s}, a_{p^{\prime}, s^{\prime}}\right]=\left[a_{p, s}^{+}, a_{p^{\prime}, s^{\prime}}^{+}\right]=0,} \\
{\left[b_{p, s}, b_{p^{\prime}, s^{\prime}}^{+}\right]=\delta_{s s^{\prime}} \delta_{p p^{\prime}}, \quad\left[b_{p, s}, b_{p^{\prime}, s^{\prime}}\right]=\left[b_{p, s}^{+}, b_{p^{\prime}, s^{\prime}}^{+}\right]=0,} \\
{\left[a_{p, s}, b_{p^{\prime}, s^{\prime}}\right]=\left[a_{p, s}, b_{p^{\prime}, s^{\prime}}^{+}\right]=\left[a_{p, s}^{+}, b_{p^{\prime}, s^{\prime}}\right]=\left[a_{p, s}^{+}, b_{p^{\prime}, s^{\prime}}^{+}\right]=0 .}
\end{gathered}
$$

It follows from commutation relations (70) that the metric is positive-definite. Thus, we construct vector space which is really a Hilbert space. For quantization of the massless fields, one needs to introduce indefinite metric (see Ref. [27]). From Eq.(68)-(70), one can find the commutation relations for different times

$$
\begin{gathered}
{\left[\Psi_{M}(x), \Psi_{N}\left(x^{\prime}\right)\right]=\left[\bar{\Psi}_{M}(x), \bar{\Psi}_{N}\left(x^{\prime}\right)\right]=0, \quad\left[\Psi_{M}(x), \bar{\Psi}_{N}\left(x^{\prime}\right)\right]=N_{M N}\left(x, x^{\prime}\right),} \\
N_{M N}\left(x, x^{\prime}\right)=N_{M N}^{+}\left(x, x^{\prime}\right)-N_{M N}^{-}\left(x, x^{\prime}\right) \\
N_{M N}^{+}\left(x, x^{\prime}\right)=\sum_{p, s}\left(\Psi_{s}^{(+)}(x)\right)_{M}\left(\bar{\Psi}_{s}^{(+)}\left(x^{\prime}\right)\right)_{N}, \\
N_{M N}^{-}\left(x, x^{\prime}\right)=\sum_{p, s}\left(\Psi_{s}^{(-)}(x)\right)_{M}\left(\bar{\Psi}_{s}^{(-)}\left(x^{\prime}\right)\right)_{N} .
\end{gathered}
$$

We obtain from Eq.(67),(71)

$$
N_{M N}^{ \pm}\left(x, x^{\prime}\right)=\sum_{p, s} \frac{m_{1}+m_{2}}{2 p_{0} V}\left(\Psi_{s}( \pm p)\right)_{M}\left(\bar{\Psi}_{s}( \pm p)\right)_{N} \exp \left[ \pm i p\left(x-x^{\prime}\right)\right] .
$$

From Eq.(27), one finds that the equation

$$
S_{(+1)}+S_{(-1)}+S_{(0)}=1,
$$

is valid. Then, from Eq.(22),(29), we obtain

$$
\Pi_{ \pm}=\sum_{s}\left(\Psi_{s}( \pm p)\right) \cdot\left(\bar{\Psi}_{s}( \pm p)\right)=\frac{ \pm i \widehat{p}\left( \pm i m_{1} \widehat{p} P \pm i m_{2} \widehat{p} \bar{P}-m_{1} m_{2}\right)}{m_{1} m_{2}\left(m_{1}+m_{2}\right)} .
$$

and Eq.(72) reads

$$
N_{M N}^{ \pm}\left(x, x^{\prime}\right)=\sum_{p} \frac{1}{2 p_{0} V}\left(\frac{ \pm i \widehat{p}\left( \pm i m_{1} \widehat{p} P \pm i m_{2} \widehat{p} \bar{P}-m_{1} m_{2}\right)}{m_{1} m_{2}}\right)_{M N} \exp \left[ \pm i p\left(x-x^{\prime}\right)\right]
$$




$$
=\left(\frac{\beta_{\mu} \partial_{\mu}\left(m_{1} \beta_{\mu} P \partial_{\mu}+m_{1} \beta_{\mu} \bar{P} \partial_{\mu}-m_{1} m_{2}\right)}{m_{1} m_{2}}\right)_{M N} \Delta_{ \pm}\left(x-x^{\prime}\right),
$$

where we introduce the singular functions [32]

$$
\Delta_{+}(x)=\sum_{p} \frac{1}{2 p_{0} V} \exp (i p x), \quad \Delta_{-}(x)=\sum_{p} \frac{1}{2 p_{0} V} \exp (-i p x) .
$$

With the aid of the function [32]

$$
\Delta_{0}(x)=i\left(\Delta_{+}(x)-\Delta_{-}(x)\right)
$$

and using Eq.(71),(75)-(77), one finds

$$
N_{M N}\left(x, x^{\prime}\right)=-i\left(\frac{\beta_{\mu} \partial_{\mu}\left(m_{1} \beta_{\mu} P \partial_{\mu}+m_{2} \beta_{\mu} \bar{P} \partial_{\mu}-m_{1} m_{2}\right)}{m_{1} m_{2}}\right)_{M N} \Delta_{0}\left(x-x^{\prime}\right) .
$$

The function $\Delta_{0}(x)$ vanishes when $x^{2}=\mathbf{x}^{2}-t^{2}>0$ 32. One can check with the help of Eq.(7), that the relation

$$
\begin{gathered}
\left(\beta_{\mu} \partial_{\mu}+m_{1} \bar{P}+m_{2} P\right) \frac{\beta_{\mu} \partial_{\mu}\left(m_{1} \beta_{\mu} P \partial_{\mu}+m_{2} \beta_{\mu} \bar{P} \partial_{\mu}-m_{1} m_{2}\right)}{m_{1} m_{2}} \\
=\frac{\left(m_{1} \bar{P}+m_{2} P\right) \beta_{\mu} \partial_{\mu}}{m_{1} m_{2}}\left(\partial_{\mu}^{2}-m_{1} m_{2}\right) .
\end{gathered}
$$

holds. Using the equation [32] $\left(\partial_{\mu}^{2}-m_{1} m_{2}\right) \Delta_{ \pm}(x)=0$, from Eq.(75),(79), we arrive at

$$
\left(\beta_{\mu} \partial_{\mu}+m_{1} \bar{P}+m_{2} P\right) N^{ \pm}\left(x, x^{\prime}\right)=0 .
$$

The vacuum expectation of chronological pairing of operators (the propagator) is defined as 32

$$
\left\langle T \Psi_{M}(x) \bar{\Psi}_{N}(y)\right\rangle_{0}=N_{M N}^{c}(x-y)
$$




$$
=\theta\left(x_{0}-y_{0}\right) N_{M N}^{+}(x-y)+\theta\left(y_{0}-x_{0}\right) N_{M N}^{-}(x-y),
$$

where $\theta(x)$ is the theta-function. Then, one obtains from Eq.(81) the propagator:

$$
\langle T \Psi(x) \cdot \bar{\Psi}(y)\rangle_{0}=\frac{\beta_{\mu} \partial_{\mu}\left(m_{1} \beta_{\mu} P \partial_{\mu}+m_{2} \beta_{\mu} \bar{P} \partial_{\mu}-m_{1} m_{2}\right)}{m_{1} m_{2}} \Delta_{c}(x-y),
$$

where

$$
\Delta_{c}(x-y)=\theta\left(x_{0}-y_{0}\right) \Delta_{+}(x-y)+\theta\left(y_{0}-x_{0}\right) \Delta_{-}(x-y)
$$

With the help of the equation [32] $\left(\partial_{\mu}^{2}-m_{1} m_{2}\right) \Delta_{c}(x)=i \delta(x)$, and from Eq.(79),(82),(83), we find

$$
\left(\beta_{\mu} \partial_{\mu}+m_{1} \bar{P}+m_{2} P\right)\langle T \Psi(x) \cdot \bar{\Psi}(y)\rangle_{0}=i \frac{m_{1} \bar{P}+m_{2} P}{m_{1} m_{2}} \beta_{\mu} \partial_{\mu} \delta(x-y) .
$$

At the chose $m_{1}=m_{2}=m$, and taking into consideration Eq.(8), $P+\bar{P}=$ 1, the propagator (82) and Eq.(84) are simplified. The above equations are not valid for the massless case, $m_{1}=0$, because of singularities in Eq.(78),(79),(82),84). For the massive case, the propagator (82) can be used for calculations of quantum processes with vector particles in the first-order formalism. The difficulty to quantize massless fields in the 10-component form of equations is connected with the fact that eigenvalues of the operator $\Lambda_{ \pm}^{(0)}$, Eq.(31), are degenerated. To avoid this difficulty, one needs to introduce a general gauge and to use the 11-component RWE. Quantization of massless fields (in the general gage) in the first-order formalism was performed in Ref. [27].

\section{Conclusion}

We have considered the massive and massless vector fields in the DKP formalism. Solutions in the form of matrix-dyads obtained allow us to make quantum-electrodynamics calculations of processes with vector particles in the covariant form. After the exclusion of the non-dynamical components the Hamiltonian forms of equations for massive and massless fields are obtained. One may consider particles in external electromagnetic fields at the level of 
the Hamiltonian form. The canonical and symmetrical Belinfante energymomentum tensors found possess their nonzero traces. We investigate the dilatation symmetry in the first-order formalism. It was demonstrated that the dilatation symmetry is broken in the massive case but in the massless case the modified dilatation current is conserved. The canonical quantization is considered in the DKP form and the propagator of the massive fields is obtained. Quantization of fields and the propagator found make it possible to use the perturbation theory for different quantum calculations in a simple manner.

\section{Appendix}

Now, we clarify the physical meaning of parameters $m_{1}, m_{2}$ introduced in Eq.(1). Eliminating the antisymmetric tensor in Eq.(1), one obtains an equation as follows:

$\left(\delta_{\mu \nu} \partial_{\alpha}^{2}-\partial_{\mu} \partial_{\nu}\right) \psi_{\nu}-m_{1} m_{2} \psi_{\mu}=0$

Let us consider the momentum space, $\partial_{\mu} \rightarrow i p_{\mu}$. Then Eq.(A1) becomes the matrix equation

$M_{\mu \nu} \psi_{\nu}=0$

where the matrix of the equation is given by

$M_{\mu \nu}=\delta_{\mu \nu}\left(p^{2}+m_{1} m_{2}\right)-p_{\mu} p_{\nu}$,

where $p^{2}=p_{\mu}^{2}=\mathbf{p}^{2}-p_{0}^{2}$. One can verify that the matrix $M=\left\{M_{\mu \nu}\right\}$ obeys the "minimal" polynomial equation

$\left(M-p^{2}-m_{1} m_{2}\right)\left(M-m_{1} m_{2}\right)=0$.

It follows from Eq.(A4) that the matrix $M$ has the eigenvalues $\lambda_{1}=p^{2}+m_{1} m_{2}$ and $\lambda_{1}=m_{1} m_{2}$. The algebraic matrix equation (A2) possesses non-trivial solutions if $\operatorname{det} M=0$. This leads to $\lambda_{1}=0$ that is equivalent to the dispersion equation

$p_{0}^{2}=\mathbf{p}^{2}+m_{1} m_{2}$,

or $\lambda_{2}=0$. Eq.(A5) tells us that the mass of a particle is $m=\sqrt{m_{1} m_{2}}$. The equation $\lambda_{2}=0$ is satisfied if $m_{1}=0$ (we do not discuss the trivial 
case $\left.m_{2}=0\right)$. Equation $m_{1}=0$ corresponds to massless case because it leads to $p_{0}^{2}=\mathbf{p}^{2}$. It should be noted that Eq.(A1) (or system (1)) does not describe simultaneously two particles (massive and massless). Eq.(A1) describes massive particles if $m_{1} \neq 0, m_{2} \neq 0$ or massless particles if $m_{1}=0$, $m_{2} \neq 0$. Thus, we have the convenient parametrization which allows us to consider both cases: massive and massless. Applying the 4-divergence to Eq.(A1), one finds that the equation $\partial_{\mu} \psi_{\mu}=0$ holds automatically only for massive case $m_{1} \neq 0, m_{2} \neq 0$. The equation $\partial_{\mu} \psi_{\mu}=0$ leaves only three physical degrees from 4 -vector $\psi_{\mu}$ corresponding to three spin projections of vector particles. For massless fields $\left(m_{1}=0\right)$ the Lorentz condition $\partial_{\mu} \psi_{\mu}=0$ does not follow from the equation of motion (A1) which becomes the wave equation for the four-potential [35].

\section{References}

[1] Gerard 't Hooft, M. J. G. Veltman, Nucl. Phys. B44, 189 (1972).

[2] G. Petiau, University of Paris thesis (1936).

[3] R. J. Duffin, Phys. Rev. 54, 1114 (1938).

[4] N. Kemmer, Proc. Roy. Soc. A173, 91 (1939).

[5] H. Umezawa, Quantum Field Theory (North-Holland, 1956).

[6] R. A. Krajcik and M. M. Nieto, Am. J. Phys. 45, 818 (1977).

[7] S. I. Kruglov, Annales Fond. Broglie 29, 1005 (2004) [Erratum-ibid 31, 489 (2006)] |arXiv:quant-ph/0408056.

[8] S. I. Kruglov, Annales Fond. Broglie 31, 343,(2006) arXiv:hep-th/0606128.

[9] S. I. Kruglov, Can. J. Phys. 85, 887 (2007) arXiv:hep-ph/0507027.

[10] S. I. Kruglov, J. Phys. A43, 245403 (2010) [arXiv:0907.1706 [hep-th]].

[11] S. I. Kruglov, Int. J. Mod. Phys. A16, 4925 (2001) arXiv:hep-th/0110083. 
[12] S. I. Kruglov, Int. J. Mod. Phys. A21, 1143 (2006) arXiv:hep-th/0405088.

[13] S. I. Kruglov, Mod. Phys. Lett. A23, 2141 (2008) arXiv:0802.0256 [hepth]].

[14] S. I. Kruglov, Int. J. Theor. Phys. 41, 653, (2002) arXiv:hep-th/0110251.

[15] S. I. Kruglov, Eur. Phys. J. C68, 337 (2010) arXiv:0911.0442 [hep-th]].

[16] S. I. Kruglov, Symmetry and Electromagnetic Interaction of Fields with Multi-Spin (Nova Science Publishers, Huntington, New York, (2001)).

[17] F. I. Fedorov, Doklady Acad. Sci. USSR 179, 802 (1968).

[18] R. Casana, J. T. Lunardi, B. M. Pimentel, R. G. Teixeira, Gen. Rel. Grav. 34, 1941 (2002) arXiv:gr-qc/0203068.

[19] R. Casana, V. Y. Fainberg, J. T. Lunardi, B. M. Pimentel, R. G. Teixeira, Class. Quant. Grav. 20, 2457 (2003) arXiv:gr-qc/0209083.

[20] A. A. Bogush, V. V. Kisel, N. G. Tokarevskaya, V. M. Red'kov, Annales Fond. Broglie 32, 355 (2007).

[21] V. Gribov, Eur. Phys. J. C10, 71 (1999) arXiv:hep-ph/9807224.

[22] Harish-Chandra, Proc. Roy. Soc. A186, 502 (1946).

[23] L. G. Moroz and F. I. Fedorov, Proc. of Institute of Physics and Mathematics, Acad. Nauk BSSR, 3, 154 (1959) (in Russian).

[24] J. T. Lunardi, B. M. Pimentel, R. G. Teixeira, J. S. Valverde, Phys. Lett. A268, 165 (2000) arXiv:hep-th/9911254].

[25] F. I. Fedorov, Sov. Phys. - JETP 35(8), 339 (1959) (Zh. Eksp. Teor. Fiz. 35, 493 (1958)).

[26] S. I. Kruglov, Annales Fond. Broglie 26, $725 \quad$ (2001) arXiv:math-ph/0110008.

[27] S. I. Kruglov, Can. J. Phys. 86, 995 (2008) [Erratum-ibid. 89, 249 (2011)] arXiv:hep-th/0610202. 
[28] P. Ghose, Foundations of Physics 26, 1441 (1996).

[29] L. Oliver, Anales de Fisica, 64, 407 (1968).

[30] M. Nowakowski, Phys. Lett. A244, 329 (1998) arXiv:quant-ph/9801020.

[31] T. R. Cardoso, L. B. Castro and A. S. de Castro, Phys. Lett. A 372, 5964 (2008) [arXiv:0806.0359 [hep-th]].

[32] A. I. Ahieser and V. B. Berestetskii, Quantum Electrodynamics (New York: Wiley Interscience, 1969).

[33] S. Coleman and R. Jakiw, Ann. Phys. 67, 552 (1971).

[34] R. Casana, B. M. Pimentel, J. T. Lunardi, R. G. Teixeira, Class. Quantum Grav. 22, 3083 (2005) arXiv:gr-qc/0311042].

[35] C. Itzykson and J. B. Zuber, Quantum Field Theory (McGraw-Hill Inc., 1980). 